\title{
A Novel Model to Design the Equilibrium Slag Compositions for Bearing Steel: Verification and Application
}

\author{
Yunpeng WANG, ${ }^{1 * *}$ Guoguang $\mathrm{CHENG}^{1}{ }^{1)}$ Shijian $\mathrm{LI}^{1)}{ }^{1)}$ Weixing DAI, ${ }^{1)}$ Dajun SHANG ${ }^{2)}$ and Chunguang $\mathrm{LU}^{2)}$ \\ 1) State Key Laboratory of Advanced Metallurgy, University of Science and Technology Beijing, 30 Xueyuan Road, Haidian \\ District, Beijing, 100083 P. R. China. \\ 2) Research and Development Department, Xining Special Steel Co., Ltd., Xining, Qinghai, 810005 China.
}

(Received on November 13, 2020; accepted on January 12, 2021)

\begin{abstract}
A new equilibrium calculation model for the slag composition design of bearing steel is established through the combination between IMCT and FactSage software. With this model, the equilibrium slag could be obtained quantitatively when the initial compositions of steel were given. It is validated by the comparison of predictive results with experimental data with different slag system, reflecting the reliability of the model. After that, the effects of $\mathrm{Al}$ in steel as well as $\mathrm{CaO}, \mathrm{MgO}$ and $\mathrm{Al}_{2} \mathrm{O}_{3}$ in slag on the dissolved oxygen, magnesium and calcium were discussed in detail. Furthermore, Industrial trials with five different slags was carried out in a special steel plant in China. Results show a good correlation between the calculated contents in molten steel after LF refining and number density of oxide inclusions in final steel, which means this model have a good guidance for the production of high clean bearing steel.
\end{abstract}

KEY WORDS: thermodynamic model; bearing steel; slag design; equilibrium slag.

\section{Introduction}

The main factors affecting the rolling contact fatigue (RCF) life of bearing steel are the nonmetallic inclusions, and the compositions of slag and steel have a crucial effect on the type and properties of non-metallic inclusions, which are very important factors for reaching the desired final steel products. ${ }^{1)}$ Meanwhile, the steel composition is affected by the slags because of the steel-slag reactions. ${ }^{2}$ So it is vital to design the appropriate slag compositions, especially during the secondary refining process. For this reason, the thermodynamic model for slag composition design was established and applied in predicting the total dissolved $\mathrm{Al}, \mathrm{O}, \mathrm{Cr}$ and other components in molten steel equilibrated with slags during the secondary refining process, and results are found to agree well with measurements. ${ }^{3-8)}$

However, there are relatively larger errors between the calculation results and the measurements of trace elements such as [Ca] ([] means this element is dissolved in molten steel). This is because the first- and second-order interaction parameters of $\mathrm{Ca}$ at steelmaking temperature in Wagner equation have a great discrepancy between different investigations. For example, at $1873 \mathrm{~K}$, the value of first-order interaction parameters $\left(e_{\mathrm{Ca}}^{O}\right)$ reported by various investigators in $\mathrm{Fe}-\mathrm{O}-$ Ca system ranged from -60 to $-9000 .{ }^{9-14)}$ Combining the first- and second-order interaction parameters $\left(e_{C a}^{O} \& r_{C a}^{O}\right)$, the

\footnotetext{
* Corresponding author: E-mail: wangyunpengustb@163.com
}

calculated activity coefficient of $\mathrm{Ca}\left(\mathrm{f}_{\mathrm{Ca}}\right)$ in $\mathrm{Fe}-\mathrm{O}-\mathrm{Ca}$ system by different researchers are shown in Fig. 1. The calculation method of activity coefficient is described in Ref. 8. The calculated value of $\mathrm{Ca}$ in molten steel is closely related with the choice of the interaction parameters. Therefore, these discrepancies on the interaction parameters result in the inaccuracy of the calculated value of $\mathrm{Ca}$ in molten steel.

From the previous investigations, a very small amount of [Ca] enables the modification of the types and the morphologies of inclusions in molten steel, ${ }^{15}$ ) and the RCF life of bearing steel varied depending on the chemical composition

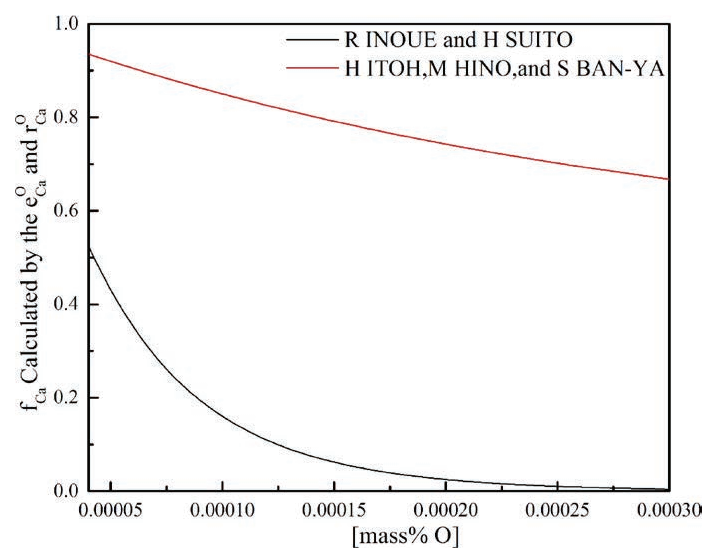

Fig. 1. The comparation of $\mathrm{f}_{\mathrm{Ca}}$ calculated by the $e_{C a}^{O}$ and $r_{C a}^{O}$ devived by different authors. (Online version in color.) 
of oxides. ${ }^{16)}$ It is beyond doubt that the calcium aluminate inclusions are most harmful to the RCF life in bearing steel because of their size and mechanical properties. Thus, it is important to establish a novel calculation model for the calculation of $\mathrm{Ca}$ elements.

Except for the interaction parameter formalism of Wagner, a thermodynamic model of metallic melts containing [O] has been established by D. Bouchard et al. ${ }^{17)}$ for the activities calculation of steel components. This model has been developed by I. H. Jung et al. ${ }^{8,18,19)}$ and has been adopted by Factsage software. Based on the thermodynamic data extracted from Factsage, the activities of trace elements such as $\mathrm{Ca}$ can be calculated in molten steel without using the first- and second-order interaction parameters. The calculation method and thermodynamic data extracted from Factsage is described in details in part 2.3. Contrast with the calculations using interaction parameters, this calculation method avoids the subjective error in choosing the interaction parameter, and the calculation results of Factsage software are widely used and credible in thermodynamic analysis. So in this model, the activities of elements in metallic melts containing $[\mathrm{O}]$ are calculated in this method.

Overall, in this work, a new equilibrium calculation model between molten steel and slag is developed through the combination of IMCT and FactSage software to lay a theoretical foundation for the design of refining slag for bearing steel. Thereafter, the equilibrium compositions, such as [O], $[\mathrm{Ca}]$ and $[\mathrm{Mg}]$ in liquid steel can be calculated, and these calculation results can be further applied in the calculation of compositions of inclusions in liquid steel. However, this paper focuses on the inclusion number density in bearing steel, so the inclusion compositions are not calculated. This model is verified by the comparison between the calculated results and measured contents in different systems. Furthermore, industrial trials are carried out, and the cleanliness of steel with different slag compositions are analyzed with this model, and the calculation results show a good guidance for the production of high clean bearing steel.

\section{Calculation Model}

\subsection{Model Schematic and Hypotheses}

Figure 2 depicts the schematic of model. The basic viewpoints and hypotheses of the model are presented as follows.

(1) This model involves three parts: the molten steel, slag, and slag-steel interface. There are various structural units in the molten steel and slag, and the equilibrium relation between molten steel and slag is established based on the reaction at the slag-steel interface.

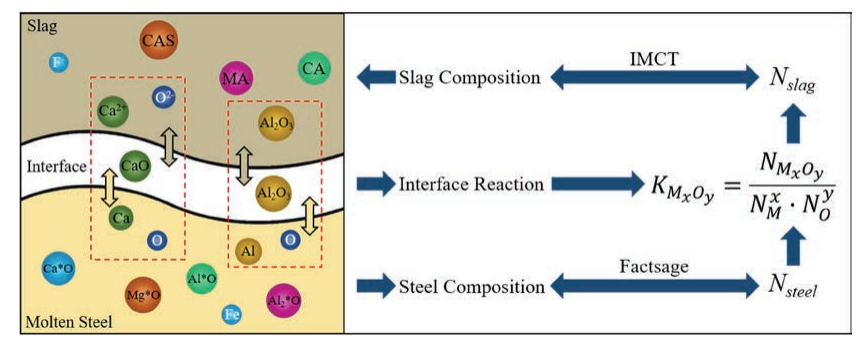

Fig. 2. The Schematic diagram of model. (Online version in color.)
(2) According to IMCT, the structural units in $\mathrm{CaO}-\mathrm{SiO}_{2}-$ $\mathrm{MgO}-\mathrm{Al}_{2} \mathrm{O}_{3}-\mathrm{CaF}_{2}$ multi-component slag are composed of simple ions, simple molecules and complex molecules; for liquid steel, this model treat it as a substitutional solution but distinguished themselves by the number of species. For example, this model assume that the species $\mathrm{Fe}, \mathrm{Al}, \mathrm{O}$, $\mathrm{Al}^{*} \mathrm{O}$, and $\mathrm{Al}_{2}{ }^{*} \mathrm{O}$ are simultaneously present in $\mathrm{Fe}-\mathrm{Al}-\mathrm{O}$ liquid system, which has been confirmed that the properties of the system can be well described by assuming these associates are present. ${ }^{17)}$ This assumption has been adopted by Factsage Software. With the help of Factsage, the equilibrium constants of these associates existing in liquid steel such as $\mathrm{Ca}^{*} \mathrm{O}, \mathrm{Mg}^{*} \mathrm{O}, \mathrm{Al}^{*} \mathrm{O}$ and $\mathrm{Al}_{2}{ }^{*} \mathrm{O}$ can be calculated out and further applied to the establishment of the model.

(3) A dynamic equilibrium occurs in both slag bulk and surface layer of liquid steel between simple ion and molecules. Both chemical reactions forming complex molecules in slag bulk and associates in liquid steel comply with mass action law. Complex molecules and associates in liquid steel do not participate in the interface reaction.

\subsection{Model for Calculating Mass Action Concentrations and Equilibrium Slag}

The distinguishing feature of IMCT is to study the thermodynamic properties of slag melt from structure characteristics. Several scholars have proved the accuracy of mass action concentration calculated by IMCT, corresponding well with the measured activities, such as in $\mathrm{CaO}-\mathrm{Al}_{2} \mathrm{O}_{3}-\mathrm{SiO}_{2}$ slags ${ }^{20)}$ and $\mathrm{CaO}-\mathrm{SiO}_{2}-\mathrm{Al}_{2} \mathrm{O}_{3}-\mathrm{MgO}$ slags. ${ }^{21)}$ In addition, this theory has been used to predict sulfur, phosphorus and manganese distribution in steelmaking successfully. ${ }^{22-25)}$ Therefore, the mass action concentration calculated by IMCT is supposed to be reliable.

According to the reported phase diagrams, ${ }^{26)}$ twenty-one complex molecules can form in $\mathrm{CaO}-\mathrm{SiO}_{2}-\mathrm{MgO}-\mathrm{Al}_{2} \mathrm{O}_{3}-$ $\mathrm{CaF}_{2}$ slag, in addition to the four simple components and two simple molecules, up to twenty-seven components. Table 1 lists all the possibly formed structural units in the multi-component slag at metallurgical temperature. The chemical reaction formulas of possibly formed complex molecules, their standard Gibbs free energy changes, mass action concentration and equilibrium constants are listed in Table 2. ${ }^{27)}$ In Table 2, the standard state of each component is liquid, and the () means this component is dissolved in liquid slag.

The mole number of components in $\mathrm{CaO}-\mathrm{SiO}_{2}-\mathrm{MgO}-$ $\mathrm{Al}_{2} \mathrm{O}_{3}-\mathrm{CaF}_{2}$ slags is assigned as $b_{1}=\Sigma n_{\mathrm{CaO}}, b_{2}=\Sigma n_{\mathrm{MgO}}$, $c_{1}=\sum n_{\mathrm{CaF}_{2}}, \quad a_{1}=\sum n_{\mathrm{Al}_{2} \mathrm{O}_{3}}, \quad a_{2}=\sum n_{\mathrm{SiO}_{2}}$ to represent chemical compositions of the slags. The equation of the mole number of components in $\mathrm{CaO}-\mathrm{SiO}_{2}-\mathrm{MgO}-\mathrm{Al}_{2} \mathrm{O}_{3}-\mathrm{CaF}_{2}$ slags is shown as follows:

$$
102 a_{1}+60 a_{2}+78 c_{1}+56 b_{1}+40 b_{2}=100
$$

The total equilibrium mole number of all structural units in $\mathrm{CaO}-\mathrm{SiO}_{2}-\mathrm{MgO}-\mathrm{Al}_{2} \mathrm{O}_{3}-\mathrm{CaF}_{2}$ slags equilibrated with molten steel $\Sigma n_{\text {slag }}$ can be expressed as follows:

$$
\sum n_{\text {slag }}=2 n_{1}+2 n_{2}+3 n_{3}+n_{4}+n_{5}+\ldots+n_{26} \ldots \ldots \ldots
$$

The mass conservation equations for components in $\mathrm{CaO}-\mathrm{SiO}_{2}-\mathrm{MgO}-\mathrm{Al}_{2} \mathrm{O}_{3}-\mathrm{CaF}_{2}$ slags equilibrated with molten steel can be established from definitions of equilibrium 
ISIJ International, Vol. 61 (2021), No. 5

Table 1. Expression of structural units of $\mathrm{CaO}-\mathrm{SiO}_{2}-\mathrm{MgO}-\mathrm{Al}_{2} \mathrm{O}_{3}$ slags at metallurgical temperature based on the IMCT.

\begin{tabular}{|c|c|c|c|}
\hline structural units in slags & $\begin{array}{l}\text { No. of structural } \\
\text { units in slags }\end{array}$ & Mole number of structural units & $\begin{array}{l}\text { Mass action concentration of } \\
\text { structural units }\end{array}$ \\
\hline$\left(\mathrm{Ca}^{2+}+\mathrm{O}^{2-}\right)$ & 1 & $n_{1}=n_{\mathrm{Ca}^{2+}, \mathrm{CaO}}=n_{\mathrm{O}^{2-}, \mathrm{CaO}}=n_{\mathrm{CaO}}$ & $N_{1}=\frac{2 n_{1}}{\sum n_{\text {slag }}}=N_{\text {CaO }}$ \\
\hline$\left(\mathrm{Mg}^{2+}+\mathrm{O}^{2-}\right)$ & 2 & $n_{2}=n_{\mathrm{Mg}^{2+}, \mathrm{MgO}}=n_{\mathrm{O}^{2-}, \mathrm{MgO}}=n_{\mathrm{MgO}}$ & $N_{2}=\frac{2 n_{2}}{\sum n_{\text {slag }}}=N_{\mathrm{MgO}}$ \\
\hline$\left(\mathrm{Ca}^{2+}+2 \mathrm{~F}^{-}\right)$ & 3 & $n_{3}=n_{\mathrm{Ca}^{2+}, \mathrm{CaF}_{2}}=0.5 \cdot n_{\mathrm{F}^{-}, \mathrm{CaF}_{2}}=n_{\mathrm{CaF}_{2}}$ & $N_{3}=\frac{3 n_{3}}{\sum n_{\text {slag }}}=N_{\mathrm{CaF}_{2}}$ \\
\hline $\mathrm{Al}_{2} \mathrm{O}_{3}$ & 4 & $n_{4}=n_{\mathrm{Al}_{2} \mathrm{O}_{3}}$ & $N_{4}=\frac{n_{4}}{\sum n_{\text {slag }}}=N_{\mathrm{Al}_{2} \mathrm{O}_{3}}$ \\
\hline $\mathrm{SiO}_{2}$ & 5 & $n_{5}=n_{\mathrm{SiO}_{2}}$ & $N_{5}=\frac{n_{5}}{\sum n_{\text {slag }}}=N_{\mathrm{SiO}_{2}}$ \\
\hline $\mathrm{CaO} \cdot \mathrm{SiO}_{2}$ & 6 & $n_{6}=n_{\mathrm{CaO} \cdot \mathrm{SiO}_{2}}$ & $N_{6}=\frac{n_{6}}{\sum n_{\text {slag }}}=N_{\mathrm{CaO} \cdot \mathrm{SiO}_{2}}$ \\
\hline $2 \mathrm{CaO} \cdot \mathrm{SiO}_{2}$ & 7 & $n_{7}=n_{2 \mathrm{CaO} \cdot \mathrm{SiO}_{2}}$ & $N_{7}=\frac{n_{7}}{\sum n_{\text {slag }}}=N_{2 \mathrm{CaO} \cdot \mathrm{SiO}_{2}}$ \\
\hline $3 \mathrm{CaO} \cdot \mathrm{SiO}_{2}$ & 8 & $n_{8}=n_{3 \mathrm{CaO} \cdot \mathrm{SiO}_{2}}$ & $N_{8}=\frac{n_{8}}{\sum n_{\text {slag }}}=N_{3 \mathrm{CaO} \cdot \mathrm{SiO}_{2}}$ \\
\hline $\mathrm{CaO} \cdot \mathrm{Al}_{2} \mathrm{O}_{3}$ & 9 & $n_{9}=n_{\mathrm{CaO} \cdot \mathrm{Al}_{2} \mathrm{O}_{3}}$ & 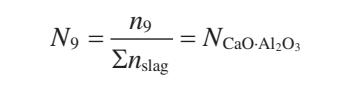 \\
\hline $\mathrm{CaO} \cdot 2 \mathrm{Al}_{2} \mathrm{O}_{3}$ & 10 & $n_{10}=n_{\mathrm{CaO} \cdot 2 \mathrm{Al}_{2} \mathrm{O}_{3}}$ & $N_{10}=\frac{n_{10}}{\sum n_{\text {slag }}}=N_{\mathrm{CaO}_{2} 2 \mathrm{Al}_{2} \mathrm{O}_{3}}$ \\
\hline $\mathrm{CaO} \cdot 6 \mathrm{Al}_{2} \mathrm{O}_{3}$ & 11 & $n_{11}=n_{\mathrm{CaO}_{6} \cdot 6 \mathrm{Al}_{2} \mathrm{O}_{3}}$ & $N_{11}=\frac{n_{11}}{\sum n_{\text {slag }}}=N_{\mathrm{CaO} 6 \mathrm{Al}_{2} \mathrm{O}_{3}}$ \\
\hline $3 \mathrm{CaO} \cdot \mathrm{Al}_{2} \mathrm{O}_{3}$ & 12 & $n_{12}=n_{3 \mathrm{CaO} \cdot \mathrm{Al}_{2} \mathrm{O}_{3}}$ & $N_{12}=\frac{n_{12}}{\sum n_{\text {slag }}}=N_{3 \mathrm{CaO} \cdot \mathrm{Al}_{2} \mathrm{O}_{3}}$ \\
\hline $12 \mathrm{CaO} \cdot 7 \mathrm{Al}_{2} \mathrm{O}_{3}$ & 13 & $n_{13}=n_{12 \mathrm{CaO} \cdot 7 \mathrm{Al}_{2} \mathrm{O}_{3}}$ & $N_{13}=\frac{n_{12}}{\sum n_{\text {slag }}}=N_{12 \mathrm{CaO} \cdot 7 \mathrm{Al}_{2} \mathrm{O}_{3}}$ \\
\hline $2 \mathrm{CaO} \cdot \mathrm{Al}_{2} \mathrm{O}_{3} \cdot \mathrm{SiO}_{2}$ & 14 & 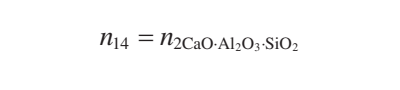 & $N_{14}=\frac{n_{14}}{\sum n_{\text {slag }}}=N_{2 \mathrm{CaO} \cdot \mathrm{Al}_{2} \mathrm{O}_{3} \cdot \mathrm{SiO}_{2}}$ \\
\hline $\mathrm{CaO} \cdot \mathrm{Al}_{2} \mathrm{O}_{3} \cdot 2 \mathrm{SiO}_{2}$ & 15 & $n_{15}=n_{\mathrm{CaO} \cdot \mathrm{Al}_{2} \mathrm{O}_{3} \cdot 2 \mathrm{SiO}_{2}}$ & $N_{15}=\frac{n_{15}}{\sum n_{\text {slag }}}=N_{\mathrm{CaO} \cdot \mathrm{Al}_{2} \mathrm{O}_{3} \cdot 2 \mathrm{SiO}_{2}}$ \\
\hline $3 \mathrm{Al}_{2} \mathrm{O}_{3} \cdot 2 \mathrm{SiO}_{2}$ & 16 & $n_{16}=n_{3 \mathrm{Al}_{2} \mathrm{O}_{3} \cdot 2 \mathrm{SiO}_{2}}$ & $N_{16}=\frac{n_{16}}{\sum n_{\text {slag }}}=N_{3 \mathrm{Al}_{2} \mathrm{O}_{3} \cdot 2 \mathrm{SiO}_{2}}$ \\
\hline $\mathrm{MgO} \cdot \mathrm{Al}_{2} \mathrm{O}_{3}$ & 17 & $n_{17}=n_{\mathrm{MgO} \cdot \mathrm{Al}_{2} \mathrm{O}_{3}}$ & 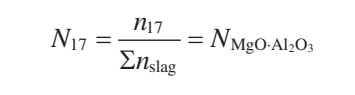 \\
\hline $\mathrm{MgO} \cdot \mathrm{SiO}_{2}$ & 18 & $n_{18}=n_{\mathrm{MgO}_{\mathrm{giO}}{ }_{2}}$ & $N_{18}=\frac{n_{18}}{\sum n_{\text {slag }}}=N_{{\mathrm{MgO} \cdot S \mathrm{Si}_{2}}}$ \\
\hline $2 \mathrm{MgO} \cdot \mathrm{SiO}_{2}$ & 19 & $n_{19}=n_{2 \mathrm{MgO} \cdot \mathrm{SiO}_{2}}$ & $N_{19}=\frac{n_{19}}{\sum n_{\text {slag }}}=N_{2 \mathrm{MgO}_{\mathrm{g}} \mathrm{SiO}_{2}}$ \\
\hline $\mathrm{CaO} \cdot \mathrm{MgO} \cdot \mathrm{SiO}_{2}$ & 20 & $n_{20}=n_{\mathrm{CaO} \cdot \mathrm{MgO} \cdot \mathrm{SiO}_{2}}$ & $N_{20}=\frac{n_{20}}{\sum n_{\text {slag }}}=N_{\mathrm{CaO} \cdot \mathrm{MgO} \cdot \mathrm{SiO}_{2}}$ \\
\hline $\mathrm{CaO} \cdot \mathrm{MgO} \cdot 2 \mathrm{SiO}_{2}$ & 21 & $n_{21}=n_{\mathrm{CaO} \cdot \mathrm{MgO} \cdot 2 \mathrm{SiO}_{2}}$ & $N_{21}=\frac{n_{21}}{\sum n_{\text {slag }}}=N_{\mathrm{CaO} \cdot M g O \cdot 2 \mathrm{SiO}_{2}}$ \\
\hline $2 \mathrm{CaO} \cdot \mathrm{MgO} \cdot 2 \mathrm{SiO}_{2}$ & 22 & 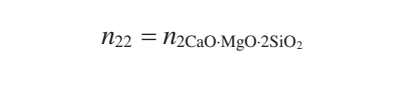 & $N_{22}=\frac{n_{22}}{\sum n_{\text {slag }}}=N_{2 \text { CaO-MgO.2.2iO }}$ \\
\hline $3 \mathrm{CaO} \cdot \mathrm{MgO} \cdot 2 \mathrm{SiO}_{2}$ & 23 & 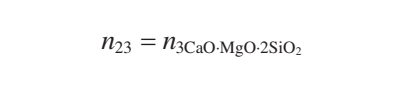 & $N_{23}=\frac{n_{23}}{\Sigma n_{\text {slag }}}=N_{3 \mathrm{CaO} \cdot \mathrm{MgO} \cdot 2 \mathrm{SiO}_{2}}$ \\
\hline $3 \mathrm{CaO} \cdot 2 \mathrm{SiO}_{2} \cdot \mathrm{CaF}_{2}$ & 24 & 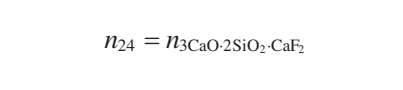 & $N_{24}=\frac{n_{24}}{\sum n_{\text {slag }}}=N_{3 \mathrm{CaO}_{2} 2 \mathrm{SiO}_{2} \cdot \mathrm{CaF}_{2}}$ \\
\hline $3 \mathrm{CaO} \cdot 3 \mathrm{Al}_{2} \mathrm{O}_{3} \cdot \mathrm{CaF}_{2}$ & 25 & 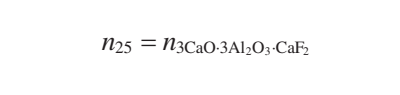 & $N_{25}=\frac{n_{25}}{\sum n_{\text {slag }}}=N_{3 \mathrm{CaO}_{3} \cdot 3 \mathrm{Al}_{2} \mathrm{O}_{3} \cdot \mathrm{CaF}_{2}}$ \\
\hline $11 \mathrm{CaO} \cdot 7 \mathrm{Al}_{2} \mathrm{O}_{3} \cdot \mathrm{CaF}_{2}$ & 26 & 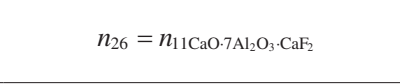 & $N_{26}=\frac{n_{26}}{\sum n_{\text {slag }}}=N_{11 \mathrm{CaO} \cdot 7 \mathrm{Al}_{2} \mathrm{O}_{3} \cdot \mathrm{CaF}_{2}}$ \\
\hline
\end{tabular}


Table 2. Chemical reaction formulas of possibly formed complex molecules, their standard molar Gibbs free energy, equilibrium constants and mass action concentrations in $\mathrm{CaO}-\mathrm{SiO}_{2}-\mathrm{MgO}-\mathrm{Al}_{2} \mathrm{O}_{3}-\mathrm{CaF}_{2}$ liquid slags at metallurgical temperature.

\begin{tabular}{|c|c|c|}
\hline Reactions & $\Delta_{r} G_{i}^{\theta}(\mathrm{J} / \mathrm{mol})$ & $N_{i}$ \\
\hline$\left(\mathrm{Ca}^{2+}+\mathrm{O}^{2-}\right)+\left(\mathrm{SiO}_{2}\right)=\left(\mathrm{CaO} \cdot \mathrm{SiO}_{2}\right)$ & $-81416-10.498 T$ & $N_{5}=K_{1} \cdot N_{1} \cdot N_{5}$ \\
\hline $2\left(\mathrm{Ca}^{2+}+\mathrm{O}^{2-}\right)+\left(\mathrm{SiO}_{2}\right)=\left(2 \mathrm{CaO} \cdot \mathrm{SiO}_{2}\right)$ & $-160431+4.106 T$ & $N_{6}=K_{2} \cdot N_{1}^{2} \cdot N_{5}$ \\
\hline $3\left(\mathrm{Ca}^{2+}+\mathrm{O}^{2-}\right)+\left(\mathrm{SiO}_{2}\right)=\left(3 \mathrm{CaO} \cdot \mathrm{SiO}_{2}\right)$ & $-93366-23.03 T$ & $N_{7}=K_{3} \cdot N_{1}^{3} \cdot N_{5}$ \\
\hline$\left(\mathrm{Ca}^{2+}+\mathrm{O}^{2-}\right)+\left(\mathrm{Al}_{2} \mathrm{O}_{3}\right)=\left(\mathrm{CaO} \cdot \mathrm{Al}_{2} \mathrm{O}_{3}\right)$ & $-18120-18.62 T$ & $N_{8}=K_{4} \cdot N_{1} \cdot N_{4}$ \\
\hline$\left(\mathrm{Ca}^{2+}+\mathrm{O}^{2-}\right)+2\left(\mathrm{Al}_{2} \mathrm{O}_{3}\right)=\left(\mathrm{CaO} \cdot 2 \mathrm{Al}_{2} \mathrm{O}_{3}\right)$ & $-16400-26.8 T$ & $N_{9}=K_{5} \cdot N_{1} \cdot N_{4}^{2}$ \\
\hline$\left(\mathrm{Ca}^{2+}+\mathrm{O}^{2-}\right)+6\left(\mathrm{Al}_{2} \mathrm{O}_{3}\right)=\left(\mathrm{CaO} \cdot 6 \mathrm{Al}_{2} \mathrm{O}_{3}\right)$ & $-17430-37.2 T$ & $N_{10}=K_{6} \cdot N_{1} \cdot N_{4}^{6}$ \\
\hline $3\left(\mathrm{Ca}^{2+}+\mathrm{O}^{2-}\right)+\left(\mathrm{Al}_{2} \mathrm{O}_{3}\right)=\left(3 \mathrm{CaO} \cdot \mathrm{Al}_{2} \mathrm{O}_{3}\right)$ & $-17000-37.0 T$ & $N_{11}=K_{7} \cdot N_{1}^{3} \cdot N_{4}$ \\
\hline $12\left(\mathrm{Ca}^{2+}+\mathrm{O}^{2-}\right)+7\left(\mathrm{Al}_{2} \mathrm{O}_{3}\right)=\left(12 \mathrm{CaO} \cdot 7 \mathrm{Al}_{2} \mathrm{O}_{3}\right)$ & $-86100-205.1 T$ & $N_{12}=K_{8} \cdot N_{1}^{12} \cdot N_{4}^{7}$ \\
\hline $2\left(\mathrm{Ca}^{2+}+\mathrm{O}^{2-}\right)+\left(\mathrm{Al}_{2} \mathrm{O}_{3}\right)+\left(\mathrm{SiO}_{2}\right)=\left(2 \mathrm{CaO} \cdot \mathrm{Al}_{2} \mathrm{O}_{3} \cdot \mathrm{SiO}_{2}\right)$ & $-61964.64-60.29 T$ & $N_{13}=K_{9} \cdot N_{1}^{2} \cdot N_{4} \cdot N_{5}$ \\
\hline$\left(\mathrm{Ca}^{2+}+\mathrm{O}^{2-}\right)+\left(\mathrm{Al}_{2} \mathrm{O}_{3}\right)+2\left(\mathrm{SiO}_{2}\right)=\left(\mathrm{CaO} \cdot \mathrm{Al}_{2} \mathrm{O}_{3} \cdot 2 \mathrm{SiO}_{2}\right)$ & $-13816.44-55.266 T$ & $N_{14}=K_{10} \cdot N_{1} \cdot N_{4} \cdot N_{5}^{2}$ \\
\hline $3\left(\mathrm{Al}_{2} \mathrm{O}_{3}\right)+2\left(\mathrm{SiO}_{2}\right)=3\left(\mathrm{Al}_{2} \mathrm{O}_{3} \cdot 2 \mathrm{SiO}_{2}\right)$ & $-4354.27-10.467 T$ & $N_{15}=K_{11} \cdot N_{4}^{3} \cdot N_{5}^{2}$ \\
\hline$\left(\mathrm{Mg}^{2+}+\mathrm{O}^{2-}\right)+\left(\mathrm{Al}_{2} \mathrm{O}_{3}\right)=\left(\mathrm{MgO} \cdot \mathrm{Al}_{2} \mathrm{O}_{3}\right)$ & $-35530-2.09 T$ & $N_{16}=K_{12} \cdot N_{2} \cdot N_{4}$ \\
\hline$\left(\mathrm{Mg}^{2+}+\mathrm{O}^{2-}\right)+\left(\mathrm{SiO}_{2}\right)=\left(\mathrm{MgO} \cdot \mathrm{SiO}_{2}\right)$ & $43400-40.0 T$ & $N_{17}=K_{13} \cdot N_{2} \cdot N_{5}$ \\
\hline $2\left(\mathrm{Mg}^{2+}+\mathrm{O}^{2-}\right)+\left(\mathrm{SiO}_{2}\right)=\left(2 \mathrm{MgO} \cdot \mathrm{SiO}_{2}\right)$ & $-77403+11.0 T$ & $N_{18}=K_{14} \cdot N_{2}^{2} \cdot N_{5}$ \\
\hline$\left(\mathrm{Ca}^{2+}+\mathrm{O}^{2-}\right)+\left(\mathrm{Mg}^{2+}+\mathrm{O}^{2-}\right)+\left(\mathrm{SiO}_{2}\right)=\left(\mathrm{CaO} \cdot \mathrm{MgO} \cdot \mathrm{SiO}_{2}\right)$ & $-124766.6+3.768 T$ & $N_{19}=K_{15} \cdot N_{1} \cdot N_{2} \cdot N_{5}$ \\
\hline$\left(\mathrm{Ca}^{2+}+\mathrm{O}^{2-}\right)+\left(\mathrm{Mg}^{2+}+\mathrm{O}^{2-}\right)+2\left(\mathrm{SiO}_{2}\right)=\left(\mathrm{CaO} \cdot \mathrm{MgO} \cdot 2 \mathrm{SiO}_{2}\right)$ & $-80387-51.916 T$ & $N_{20}=K_{16} \cdot N_{1} \cdot N_{2} \cdot N_{5}^{2}$ \\
\hline $2\left(\mathrm{Ca}^{2+}+\mathrm{O}^{2-}\right)+\left(\mathrm{Mg}^{2+}+\mathrm{O}^{2-}\right)+2\left(\mathrm{SiO}_{2}\right)=\left(2 \mathrm{CaO} \cdot \mathrm{MgO} \cdot 2 \mathrm{SiO}_{2}\right)$ & $-73688-63.639 T$ & $N_{21}=K_{17} \cdot N_{1}^{2} \cdot N_{2} \cdot N_{5}^{2}$ \\
\hline $3\left(\mathrm{Ca}^{2+}+\mathrm{O}^{2-}\right)+\left(\mathrm{Mg}^{2+}+\mathrm{O}^{2-}\right)+2\left(\mathrm{SiO}_{2}\right)=3\left(\mathrm{CaO} \cdot \mathrm{MgO} \cdot 2 \mathrm{SiO}_{2}\right)$ & $-315469+24.786 T$ & $N_{22}=K_{18} \cdot N_{1}^{3} \cdot N_{2} \cdot N_{5}^{2}$ \\
\hline $3\left(\mathrm{Ca}^{2+}+\mathrm{O}^{2-}\right)+2\left(\mathrm{SiO}_{2}\right)+\left(\mathrm{Ca}^{2+}+2 \mathrm{~F}^{-}\right)=\left(3 \mathrm{CaO} \cdot 2 \mathrm{SiO}_{2} \cdot \mathrm{CaF}_{2}\right)$ & $-255180-8.2 T$ & $N_{23}=K_{19} \cdot N_{1}^{3} \cdot N_{2}^{2} \cdot N_{3}$ \\
\hline $3\left(\mathrm{Ca}^{2+}+\mathrm{O}^{2-}\right)+3\left(\mathrm{Al}_{2} \mathrm{O}_{3}\right)+\left(\mathrm{Ca}^{2+}+2 \mathrm{~F}^{-}\right)=\left(3 \mathrm{CaO} \cdot 3 \mathrm{Al}_{2} \mathrm{O}_{3} \cdot \mathrm{CaF}_{2}\right)$ & $-44492-73.15 T$ & $N_{24}=K_{20} \cdot N_{1}^{3} \cdot N_{4}^{3} \cdot N_{3}$ \\
\hline $11\left(\mathrm{Ca}^{2+}+\mathrm{O}^{2-}\right)+7\left(\mathrm{Al}_{2} \mathrm{O}_{3}\right)+\left(\mathrm{Ca}^{2+}+2 \mathrm{~F}^{-}\right)=\left(11 \mathrm{CaO} \cdot 7 \mathrm{Al}_{2} \mathrm{O}_{3} \cdot \mathrm{CaF}_{2}\right)$ & $-228760-155.8 T$ & $N_{25}=K_{21} \cdot N_{1}^{11} \cdot N_{4}^{7} \cdot N_{3}$ \\
\hline
\end{tabular}

mole numbers $n_{i}$ and mass action concentrations $N_{i}$ of all structural units listed in Tables 1 and 2 as follows:

$$
\begin{aligned}
& a_{1}=\Sigma n_{\text {slag }}\left(N_{4}+N_{9}+2 N_{10}+6 N_{11}+N_{12}\right. \\
&\left.+7 N_{13}+N_{14}+N_{15}+3 N_{16}+N_{17}+3 N_{25}+7 N_{26}\right) \\
& a_{2}=\Sigma n_{\text {slag }}\left(N_{5}+N_{6}+N_{7}+N_{8}+N_{14}+2 N_{15}\right. \\
&+2 N_{16}+N_{18}+N_{19}+N_{20}+2 N_{21}+2 N_{22} \ldots \\
&\left.+2 N_{23}+2 N_{24}\right) \\
& b_{1}=\Sigma n_{\text {slag }}\left(0.5 N_{1}+1 / 3 N_{3}+N_{6}+2 N_{7}+3 N_{8}\right. \\
&+N_{9}+N_{10}+N_{11}+3 N_{12}+12 N_{13}+2 N_{14} \\
&+ N_{15}+N_{20}+N_{21}+2 N_{22}+3 N_{23}+3 N_{24} \\
&\left.+3 N_{25}+11 N_{26}\right) \\
& b_{2}= \sum n_{\text {slag }}\left(0.5 N_{2}+N_{17}+N_{18}+2 N_{19}+N_{20} \ldots .\right. \\
&\left.+N_{21}+N_{22}+N_{23}\right) \\
& c_{1}=\Sigma n_{\text {slag }}\left(1 / 3 N_{3}+N_{24}+N_{25}+N_{26}\right) \ldots \ldots . . .
\end{aligned}
$$

Besides, according to the principle that the sum of mole fraction for all structural units in $\mathrm{CaO}-\mathrm{SiO}_{2}-\mathrm{MgO}-\mathrm{Al}_{2} \mathrm{O}_{3}-$ $\mathrm{CaF}_{2}$ slags with a fixed amount under equilibrium condition is equal to 1 , the following expression can be obtained:

$$
\sum_{i=1}^{26} \mathrm{~N}_{\mathrm{i}}=1
$$

Hence, the governing equation group of this thermodynamic model for calculating mass action concentrations of structural units in $\mathrm{CaO}-\mathrm{SiO}_{2}-\mathrm{MgO}-\mathrm{Al}_{2} \mathrm{O}_{3}-\mathrm{CaF}_{2}$ slags equilibrated with molten steel at metallurgical temperature can be established by Eqs. (1)-(8) and Tables 1-2. ${ }^{27)}$

\subsection{Model for Calculating Activities of Structural Units in Molten Steels}

According to Factsage 7.0 software, the equilibrium constants $\mathrm{K}$ of $\mathrm{M}^{*} \mathrm{O}$ and $\mathrm{M}_{2}{ }^{*} \mathrm{O}$ can be obtained through the Phase Diagram Module by selecting the Foxide and Fsteel data base, then the standard molar Gibbs free energy of each reaction at a given temperature can be calculated. ${ }^{28)}$

The equilibrium constants of $\mathrm{O}$-containing structural units in $\mathrm{Al}-\mathrm{O}-\mathrm{Fe}, \mathrm{Si}-\mathrm{O}-\mathrm{Fe}, \mathrm{Ca}-\mathrm{O}-\mathrm{Fe}$, and $\mathrm{Mg}-\mathrm{O}-\mathrm{Fe}$ systems in molten steel is calculated by Factsage, the $\mathrm{Mn}-\mathrm{O}-\mathrm{Fe}$ and $\mathrm{Cr}-\mathrm{O}-\mathrm{Fe}$ systems are also calculated because $\mathrm{Mn}$ and $\mathrm{Cr}$ are essential components in bearing steel. The results are shown in Table 3. In this study, the equilibrium constants $\mathrm{K}$ is calculated at $1733 \mathrm{~K}, 1793 \mathrm{~K}, 1833 \mathrm{~K}$ and $1873 \mathrm{~K}$, and the calculation results of other temperatures can be obtained by selecting the Foxide and Fsteel data base and changing the 
Table 3. Chemical reaction formulas of O-containing structural units and equilibrium constants in molten steel at different metallurgical temperatures.

\begin{tabular}{ccccc}
\hline \multirow{2}{*}{ Reaction } & \multicolumn{4}{c}{ K at different metallurgical temperatures } \\
\cline { 2 - 5 } & $1873 \mathrm{~K}$ & $1833 \mathrm{~K}$ & $1793 \mathrm{~K}$ & $1733 \mathrm{~K}$ \\
\hline$[\mathrm{Al}]+[\mathrm{O}]=\left[\mathrm{Al}^{*} \mathrm{O}\right]$ & $6.26 \times 10^{2}$ & $7.11 \times 10^{2}$ & $9.60 \times 10^{2}$ & $1.20 \times 10^{3}$ \\
$2[\mathrm{Al}]+[\mathrm{O}]=\left[\mathrm{Al}_{2}{ }^{*} \mathrm{O}\right]$ & $3.58 \times 10^{4}$ & $4.39 \times 10^{4}$ & $7.58 \times 10^{4}$ & $1.08 \times 10^{5}$ \\
{$[\mathrm{Si}]+[\mathrm{O}]=\left[\mathrm{Si}^{*} \mathrm{O}\right]$} & 3.84 & 3.81 & 3.91 & 5.85 \\
{$[\mathrm{Ca}]+[\mathrm{O}]=\left[\mathrm{Ca}^{*} \mathrm{O}\right]$} & $4.49 \times 10^{8}$ & $5.59 \times 10^{8}$ & $6.00 \times 10^{8}$ & $8.48 \times 10^{8}$ \\
{$[\mathrm{Mg}]+[\mathrm{O}]=\left[\mathrm{Mg}{ }^{*} \mathrm{O}\right]$} & $7.73 \times 10^{5}$ & $8.21 \times 10^{5}$ & $8.99 \times 10^{5}$ & $9.83 \times 10^{5}$ \\
{$[\mathrm{Mn}]+[\mathrm{O}]=\left[\mathrm{Mn}{ }^{*} \mathrm{O}\right]$} & 3.21 & 3.31 & 3.03 & 3.05 \\
{$[\mathrm{Cr}]+[\mathrm{O}]=\left[\mathrm{Cr}^{*} \mathrm{O}\right]$} & $1.33 \times 10$ & $1.38 \times 10$ & $1.31 \times 10$ & $1.4 \times 10$ \\
$2[\mathrm{Cr}]+[\mathrm{O}]=\left[\mathrm{Cr}_{2}{ }^{*} \mathrm{O}\right]$ & $6.90 \times 10$ & $7.71 \times 10$ & $7.22 \times 10$ & $8.80 \times 10$ \\
\hline
\end{tabular}

set temperature in Phase Diagram Module of Factsage 7.0.

The mass action concentrations of these O-containing structural units can be calculated as follows:

$$
\begin{aligned}
& N_{\mathrm{Al}{ }^{*} \mathrm{O}}=K_{\mathrm{Al} *_{\mathrm{O}}} \cdot N_{\mathrm{Al}} \cdot N_{\mathrm{O}} \\
& N_{\mathrm{Al}_{2} * \mathrm{O}}=K_{\mathrm{Al}_{2} * \mathrm{O}} \cdot N_{\mathrm{Al}}^{2} \cdot N_{\mathrm{O}} \\
& N_{\mathrm{Si} * \mathrm{O}}=K_{\mathrm{Si} * \mathrm{O}} \cdot N_{\mathrm{Si}} \cdot N_{\mathrm{O}} \\
& N_{\mathrm{Ca} * \mathrm{O}}=K_{\mathrm{Ca} * \mathrm{O}} \cdot N_{\mathrm{Ca}} \cdot N_{\mathrm{O}} \\
& N_{\mathrm{Mg} * \mathrm{O}}=K_{\mathrm{Mg} * \mathrm{O}} \cdot N_{\mathrm{Mg}} \cdot N_{\mathrm{O}} \\
& N_{\mathrm{Mn} * \mathrm{O}}=K_{\mathrm{Mn} * \mathrm{O}} \cdot N_{\mathrm{Mn}} \cdot N_{\mathrm{O}} \\
& N_{\mathrm{Cr} * \mathrm{O}}=K_{\mathrm{Cr} * \mathrm{O}} \cdot N_{\mathrm{Cr}} \cdot N_{\mathrm{O}} \\
& N_{\mathrm{Cr}_{2} * \mathrm{O}}=K_{\mathrm{Cr}_{2} * \mathrm{O}} \cdot N_{\mathrm{Cr}}^{2} \cdot N_{\mathrm{O}}
\end{aligned}
$$

These equations between O-containing structural units and steel concentrations can be established according to the mass conservation:

$$
\begin{aligned}
& T\left[n_{\mathrm{Al}}\right]=n_{\mathrm{Al}}+n_{\mathrm{Al} * \mathrm{O}}+2 n_{\mathrm{Al}_{2} * \mathrm{O}} \\
& T\left[n_{\mathrm{Si}}\right]=n_{\mathrm{Si}}+n_{\mathrm{Si} * \mathrm{O}} \\
& T\left[n_{\mathrm{Ca}}\right]=n_{\mathrm{Ca}}+n_{\mathrm{Ca} * \mathrm{O}} \\
& T\left[n_{\mathrm{Mg}}\right]=n_{\mathrm{Mg}}+n_{\mathrm{Mg} * \mathrm{O}} \\
& T\left[n_{\mathrm{Mn}}\right]=n_{\mathrm{Mn}}+n_{\mathrm{Mn} * \mathrm{O}} \\
& T\left[n_{\mathrm{Cr}}\right]=n_{\mathrm{Cr}}+n_{\mathrm{Cr} * \mathrm{O}}+2 n_{\mathrm{Cr}_{2} * \mathrm{O}} \\
& T\left[n_{\mathrm{O}}\right]=n_{\mathrm{O}}+n_{\mathrm{Al}^{*} \mathrm{O}}+n_{\mathrm{Al}_{2} * \mathrm{O}}+n_{\mathrm{Si}^{*} \mathrm{O}}+n_{\mathrm{Ca}^{*} \mathrm{O}} \\
& +n_{\mathrm{Mg} * \mathrm{O}}+n_{\mathrm{Mn} * \mathrm{O}}+n_{\mathrm{Cr} * \mathrm{O}}+n_{\mathrm{Cr}_{2} * \mathrm{O}}
\end{aligned}
$$

Where $T\left[n_{\mathrm{M}}\right]$ is the total mole number of each metallic element in molten steel, $T\left[n_{\mathrm{O}}\right]$ is the total mole number of $\mathrm{O}$ in molten steel, $n_{\mathrm{M}}$ is the mole number of simple elements in molten steel such as $\mathrm{Al}, n_{\mathrm{M}^{*} \mathrm{O}}$ and $n_{\mathrm{M}_{2} \mathrm{O}}$ is the mole number of associates in molten steel such as $\mathrm{Al}^{*} \mathrm{O}$ and $\mathrm{Al}_{2}{ }^{*} \mathrm{O}$.
Besides, according to the principle that the sum of mole fraction for all structural units in molten steel with a fixed amount under equilibrium condition is equal to 1 , the following expression can be obtained:

$$
\begin{aligned}
& N_{\mathrm{Al}}+N_{\mathrm{Al}^{*} \mathrm{O}}+N_{\mathrm{Al}_{2} * \mathrm{O}}+N_{\mathrm{Si}}+N_{\mathrm{Si}^{*} *}+N_{\mathrm{Ca}}+N_{\mathrm{Ca}^{*} \mathrm{O}} \\
& +N_{\mathrm{Mg}}+N_{\mathrm{Mg} * \mathrm{O}}+N_{\mathrm{Mn}}+N_{\mathrm{Mn} * \mathrm{O}}+N_{\mathrm{Cr}}+N_{\mathrm{Cr} * \mathrm{O}} \\
& +N_{\mathrm{Cr}_{2} * \mathrm{O}}+N_{\mathrm{O}}+N_{\mathrm{C}}+N_{\mathrm{Fe}}=1
\end{aligned}
$$

The activities of structural units $N_{\mathrm{i}}$ is calculated as follows:

$$
N_{i}=\frac{n_{i}}{\sum n_{\text {metal }}}
$$

The $\Sigma n_{\text {metal }}$ in Eq. (24) can be calculated as follows:

$$
\begin{aligned}
\sum n_{\text {metal }}= & n_{\mathrm{Al}}+n_{\mathrm{Al} * \mathrm{O}}+n_{\mathrm{Al}_{2} * \mathrm{O}}+n_{\mathrm{Si}}+n_{\mathrm{Si} * \mathrm{O}}+n_{\mathrm{Ca}} \\
& +n_{\mathrm{Ca}^{*} \mathrm{O}}+n_{\mathrm{Mg}}+n_{\mathrm{Mg}^{*} \mathrm{O}}+n_{\mathrm{Mn}}+n_{\mathrm{Mn} * \mathrm{O}} \\
& +n_{\mathrm{Cr}}+n_{\mathrm{Cr}^{*} \mathrm{O}}+n_{\mathrm{Cr}_{2} * \mathrm{O}}+n_{\mathrm{O}}+n_{\mathrm{C}}+n_{\mathrm{Fe}}
\end{aligned}
$$

The $n_{\mathrm{C}}$ can be calculated if the [C] in molten steel is given. The method to calculate $n_{\mathrm{Fe}}$ is shown as follows:

$$
\begin{aligned}
n_{\mathrm{Fe}} & =\left(100-27 n_{\mathrm{Al}}-43 n_{\mathrm{Al} * \mathrm{O}}-70 n_{\mathrm{Al}_{2} * \mathrm{O}}-28 n_{\mathrm{Si}}\right. \\
& -44 n_{\mathrm{Si} * \mathrm{O}}-40 n_{\mathrm{Ca}}-56 n_{\mathrm{Ca} * \mathrm{O}}-24 n_{\mathrm{Mg}}-40 n_{\mathrm{Mg}^{*} \mathrm{O}} \\
& -55 n_{\mathrm{Mn}}-71 n_{\mathrm{Mn} * \mathrm{O}}-52 n_{\mathrm{Cr}}-68 n_{\mathrm{Cr} * \mathrm{O}}-120 n_{\mathrm{Cr}_{2} * \mathrm{O}} \\
& \left.-16 n_{\mathrm{O}}-[\% \mathrm{C}]\right) / 56
\end{aligned}
$$

Hence, the governing equation group of this thermodynamic model for calculating mass action concentrations of structural units in molten steel equilibrated with slags at metallurgical temperature can be established by Eqs. (9) (27) and Table $3 .^{28)}$

\subsection{Reactions between Steel and Slag at the Interface}

In the previous sections, the calculation model for slag and molten steel is established respectively. Then, the equilibrium relation between molten steel and slag is established based on the reaction between each other.

The structural units participated in the reactions between slag and molten steel are simple molecules such as $\mathrm{SiO}_{2}$ and $\mathrm{Al}_{2} \mathrm{O}_{3}$, and ionic substances such as $\left(\mathrm{Ca}^{2+}+\mathrm{O}^{2-}\right)$ and $\left(\mathrm{Mg}^{2+}+\mathrm{O}^{2-}\right)$ in slag; simple elements such as [Si] and [Al], and associates such as $\left[\mathrm{Ca}^{*} \mathrm{O}\right]$ and $\left[\mathrm{Mg}^{*} \mathrm{O}\right]$ in molten steel. At the interface, the equilibrium constants of steel-slag reactions in this model can be obtained through a fit of the calculation results of the Phase Diagram Module of FactSage. ${ }^{28)}$ The results are shown in Table 4.

According to these equations shown above, once the main compositions of molten steel $(\mathrm{C}, \mathrm{Si}, \mathrm{Mn}, \mathrm{Cr}, \mathrm{Al})$ and temperature are given, the equilibrium compositions of $\mathrm{CaO}-\mathrm{SiO}_{2}-\mathrm{MgO}-\mathrm{Al}_{2} \mathrm{O}_{3}-\mathrm{CaF}_{2}$ slag and dissolved [O], $[\mathrm{Mg}]$ and $[\mathrm{Ca}]$ in liquid steel can be obtained as long as $\left(\mathrm{CaF}_{2}\right)$ and other two contents of this multi-component slag are given. These data are input into Microsoft Excel worksheet and called by Matlab R2015b. By changing the initial data, different equilibrium slag can be obtained.

\section{Validation of Model}

A necessary step in the model development is its validation by the comparison of predictive results with experimen- 
tal data. H. Suito et al. have determined the contents of [O], $[\mathrm{Al}]$ and $[\mathrm{Ca}]$ in molten steel equilibrated with $\mathrm{CaO}-\mathrm{Al}_{2} \mathrm{O}_{3}$ binary system, as well as $\mathrm{CaO}-\mathrm{Al}_{2} \mathrm{O}_{3}-\mathrm{SiO}_{2}$ ternary system at $1873 \mathrm{~K}$ in laboratory. ${ }^{29-31)}$ This model was validated by the comparison between these measured contents and the calculated results in different slag. The results are shown in Figs. 3 and 4 respectively.

Figure 3 is the comparison between measured and calculated $[\mathrm{O}]$ and $[\mathrm{Ca}]$ content of molten steel equilibrated with

Table 4. Chemical reaction formulas of interface reactions between structural units in molten steel and slag and their equilibrium constants at different metallurgical temperatures.

\begin{tabular}{ccccc}
\hline \multirow{2}{*}{ Reaction } & \multicolumn{4}{c}{ K at different metallurgical temperatures } \\
\cline { 2 - 5 } & $1873 \mathrm{~K}$ & $1833 \mathrm{~K}$ & $1793 \mathrm{~K}$ & $1733 \mathrm{~K}$ \\
\hline $2[\mathrm{Al}]+3[\mathrm{O}]=\left(\mathrm{Al}_{2} \mathrm{O}_{3}\right)_{\text {slag }}$ & $1.08 \times 10^{21}$ & $5.31 \times 10^{21}$ & $7.61 \times 10^{22}$ & $1.31 \times 10^{24}$ \\
{$[\mathrm{Si}]+2[\mathrm{O}]=\left(\mathrm{SiO}_{2}\right)_{\text {slag }}$} & $2.84 \times 10^{9}$ & $5.99 \times 10^{9}$ & $1.43 \times 10^{10}$ & $1.04 \times 10^{11}$ \\
{$[\mathrm{Ca}]+[\mathrm{O}]=(\mathrm{CaO})_{\text {slag }}$} & $5.90 \times 10^{4}$ & $1.16 \times 10^{5}$ & $2.93 \times 10^{5}$ & $9.28 \times 10^{5}$ \\
{$[\mathrm{Mg}]+[\mathrm{O}]=(\mathrm{MgO})_{\text {slag }}$} & $5.61 \times 10^{4}$ & $1.12 \times 10^{5}$ & $2.89 \times 10^{5}$ & $9.52 \times 10^{5}$ \\
\hline
\end{tabular}

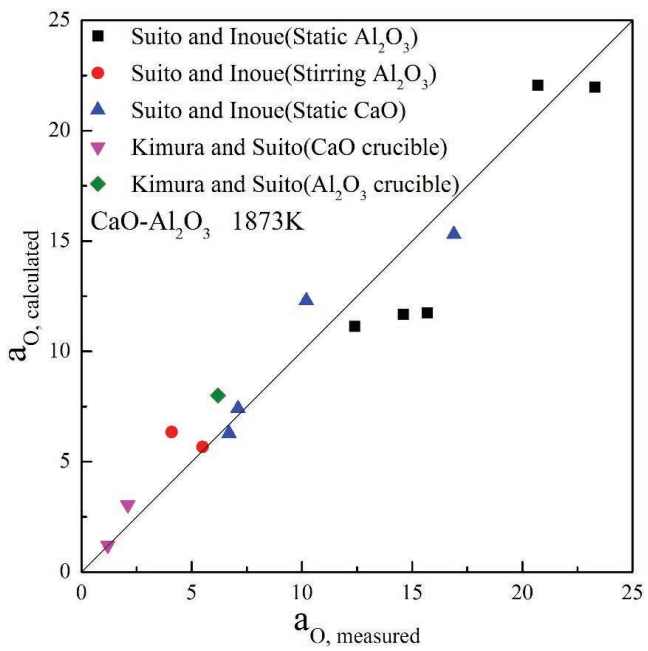

(a)

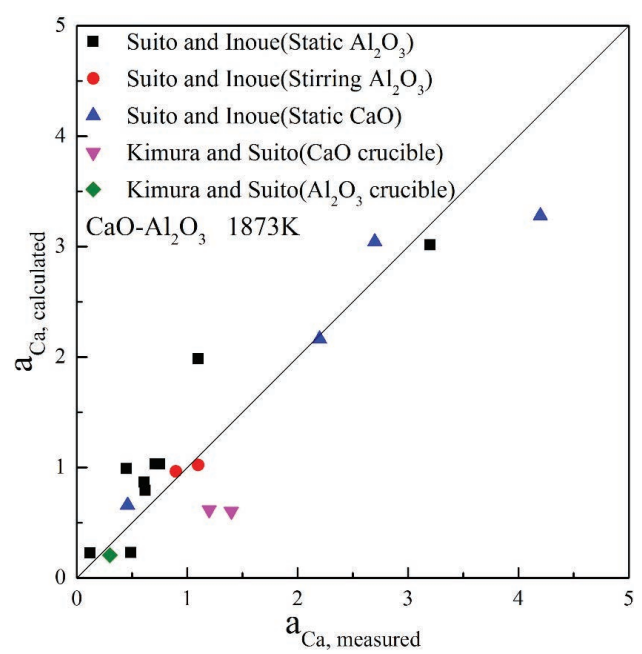

(b)

Fig. 3. Comparison between tested and calculated contents of molten steel equilibrated with $\mathrm{CaO}-\mathrm{Al}_{2} \mathrm{O}_{3}$ binary system at $1873 \mathrm{~K}$, (a) $\mathrm{O}$, (b) $\mathrm{Ca}$. (Online version in color.)
$\mathrm{CaO}-\mathrm{Al}_{2} \mathrm{O}_{3}$ binary system when soluble $[\mathrm{Al}]$ in molten steel is $0-0.03$ mass $\%$ at $1873 \mathrm{~K}$. It is shown that the calculated $[\mathrm{O}]$ and $[\mathrm{Ca}]$ are in good agreement with measured contents both at static and string conditions. However, the measured [Ca] contents are some higher than the calculated $[\mathrm{Ca}]$ in $\mathrm{CaO}$ crucible because of the crucible erosion. The comparison between measured and calculated $[\mathrm{O}]$ and $[\mathrm{Ca}]$ content of molten steel equilibrated with $\mathrm{CaO}-\mathrm{SiO}_{2}-\mathrm{Al}_{2} \mathrm{O}_{3}$ ternary system when soluble [Al] in molten steel is $0-0.03$ mass $\%$ at $1873 \mathrm{~K}$ are shown in Fig. 4. It is shown that the calculated $[\mathrm{O}]$ and $[\mathrm{Ca}]$ results are close to the measured results both with $\mathrm{Al}_{2} \mathrm{O}_{3}$ crucible and $\mathrm{CaO}$ crucible.

Concerning about $\mathrm{CaO}-\mathrm{SiO}_{2}-\mathrm{Al}_{2} \mathrm{O}_{3}-\mathrm{MgO}$ quaternary system, Kamrooz Riyahimalayeri et al. measured the oxygen activities in molten steel after vacuum degassing for 10 samples. ${ }^{32)}$ Figure 5 presents the calculated oxygen activities of the ten different sampled heats versus their respective sample numbers. As could be seen six different combinations of models including this model were applied in order to present oxygen activities of molten steel after degassing process. According to Fig. 5, it is shown that the calculated results of this work are close to the measured results com-

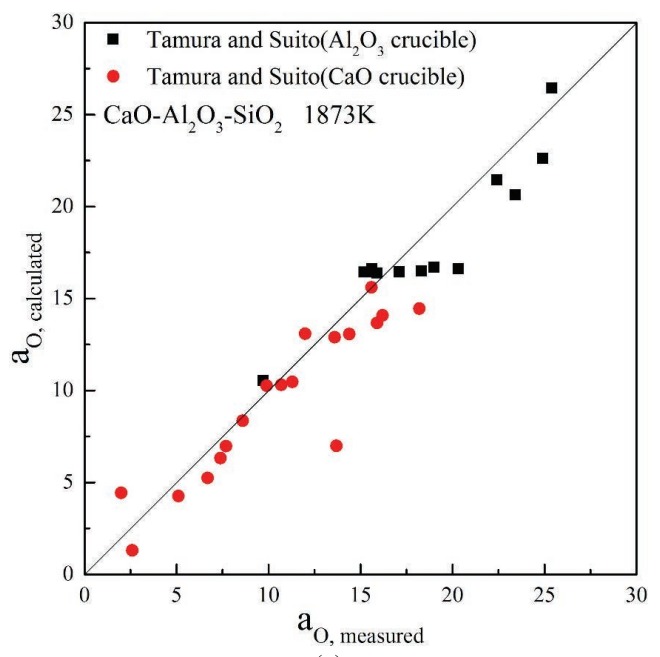

(a)

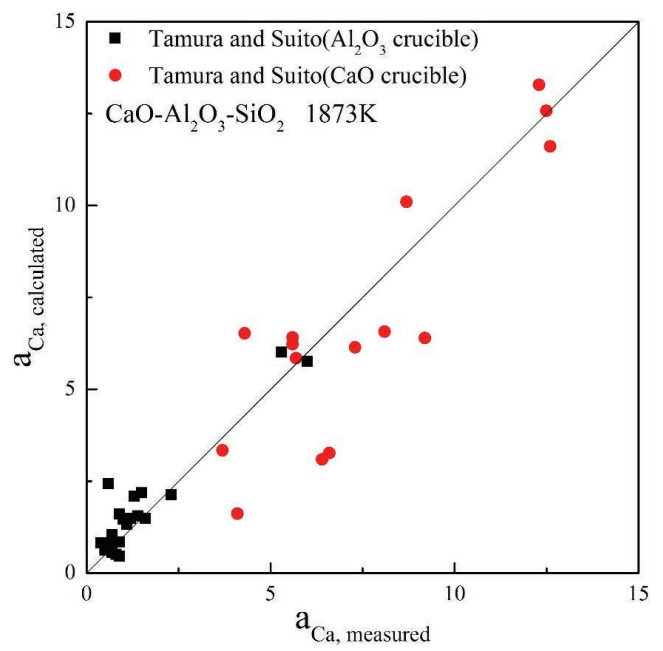

(b)

Fig. 4. Comparison between tested and calculated contents of molten steel equilibrated with $\mathrm{CaO}-\mathrm{Al}_{2} \mathrm{O}_{3}-\mathrm{SiO}_{2}$ ternary system at $1873 \mathrm{~K}$, (a) $\mathrm{O}$, (b) $\mathrm{Ca}$. (Online version in color.) 


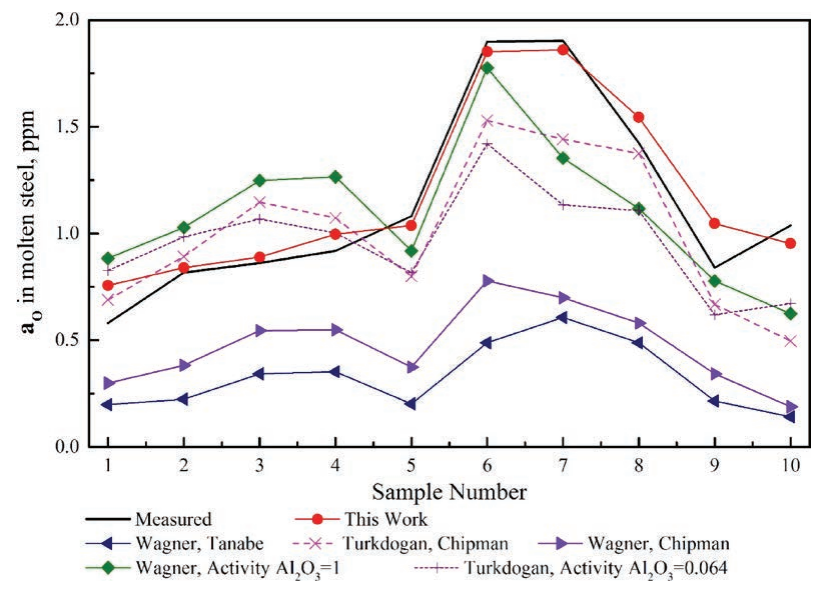

Fig. 5. The calculated and the measured oxygen activities of ten different sampled heats. (Online version in color.)

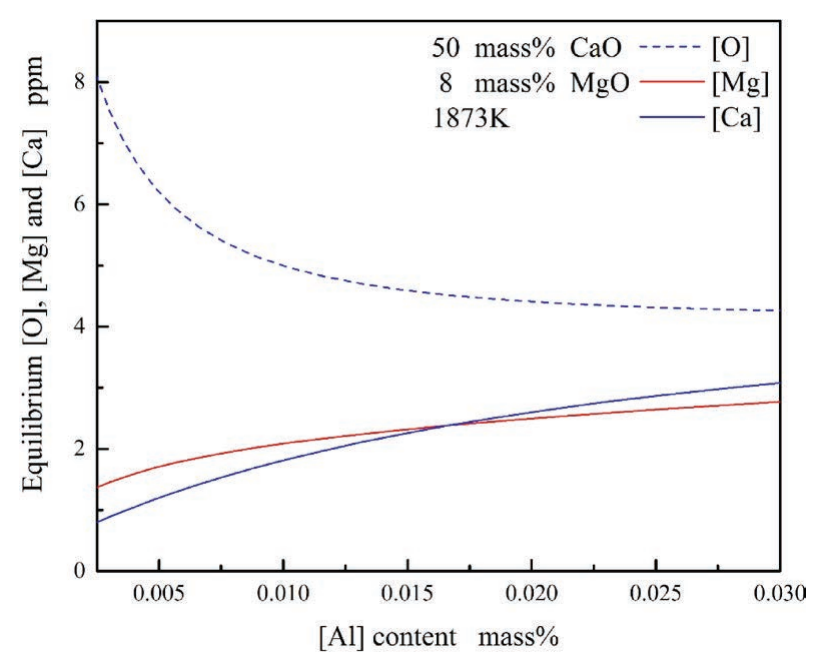

Fig. 6. Effect of Al content in molten steel on the predicted equilibrium $[\mathrm{O}],[\mathrm{Mg}]$ and $[\mathrm{Ca}]$ content. (Online version in color.)

pared with other models.

In summary, this equilibrium calculation model is reliable in predicting the composition of molten steel equilibrated with $\mathrm{CaO}-\mathrm{Al}_{2} \mathrm{O}_{3}$ binary system, $\mathrm{CaO}-\mathrm{SiO}_{2}-\mathrm{Al}_{2} \mathrm{O}_{3}$ ternary system and $\mathrm{CaO}-\mathrm{SiO}_{2}-\mathrm{Al}_{2} \mathrm{O}_{3}-\mathrm{MgO}$ quaternary system, especially when soluble [Al] in molten steel is $0-0.03$ mass $\%$. Besides, compared with other models, the calculation results of this model are closer to the measured results.

\section{Application of This Model}

The reasonability of this model has been verified through the above analysis. After that, taking GCr15 as an example, the effects of $\mathrm{Al}$ in molten steel as well as $\mathrm{CaO}, \mathrm{MgO}$ and $\mathrm{Al}_{2} \mathrm{O}_{3}$ in slag on the dissolved [O], $[\mathrm{Mg}]$ and [Ca] of slag melt are discussed in detail. The results are supposed to provide some guidance for the control of finished steel cleanliness, especially for the control of calcium aluminum inclusions.

\subsection{Effect of [Al] Content on Equilibrium $[\mathrm{O}],[\mathrm{Mg}]$ and $[\mathrm{Ca}]$}

It is of great importance for bearing steel to reasonably design the $\mathrm{Al}$ content in molten steel. Figure 6 shows the
Table 5. The main chemical compositions of GCr15, mass $\%$.

\begin{tabular}{ccccc}
\hline $\mathrm{C}$ & $\mathrm{Si}$ & $\mathrm{Mn}$ & $\mathrm{Cr}$ & $\mathrm{Al}$ \\
\hline 1 & 0.25 & 0.35 & 1.5 & $0-0.03$ \\
\hline
\end{tabular}

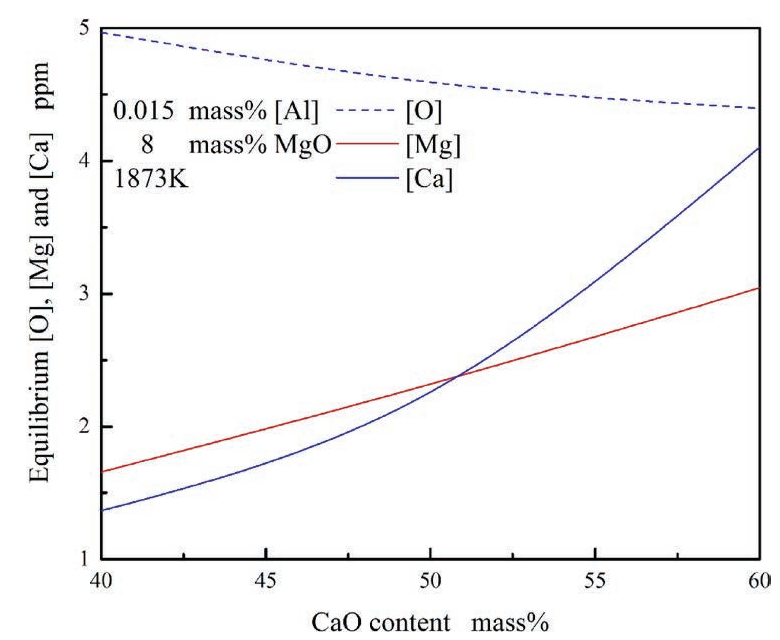

Fig. 7. Effect of $\mathrm{CaO}$ content in slag on the predicted equilibrium $[\mathrm{O}],[\mathrm{Mg}]$ and $[\mathrm{Ca}]$ content. (Online version in color.)

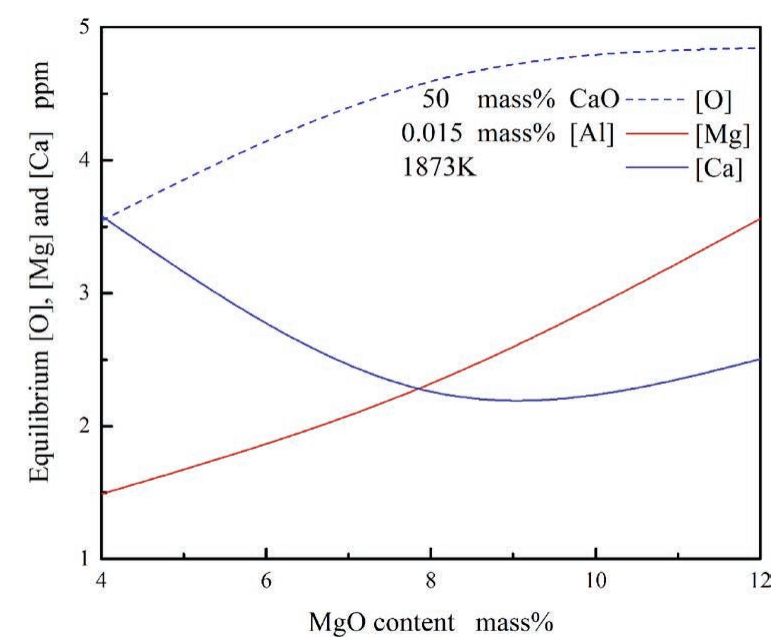

Fig. 8. Effect of $\mathrm{MgO}$ content in slag on the predicted equilibrium $[\mathrm{O}],[\mathrm{Mg}]$ and $[\mathrm{Ca}]$ content. (Online version in color.)

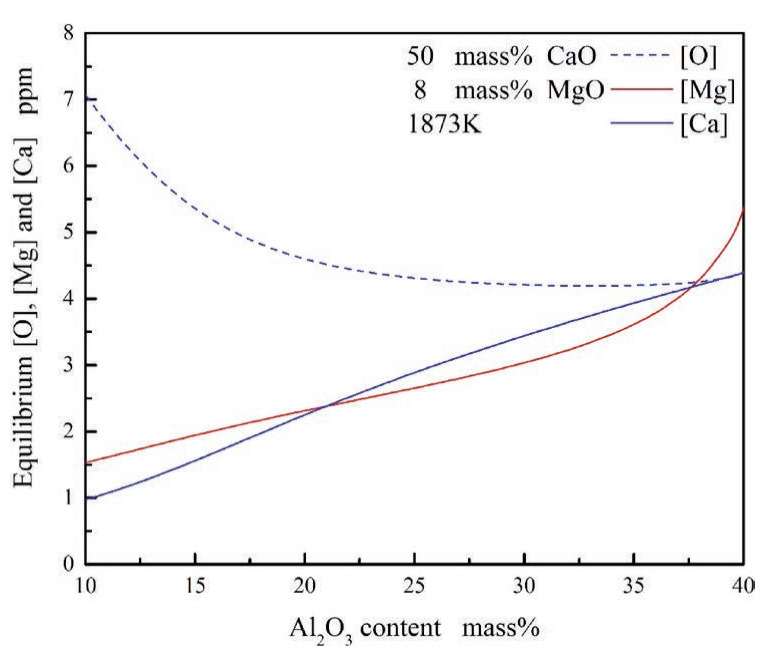

Fig. 9. Effect of $\mathrm{Al}_{2} \mathrm{O}_{3}$ content in slag on the predicted equilibrium $[\mathrm{O}],[\mathrm{Mg}]$ and $[\mathrm{Ca}]$ content. (Online version in color.) 
effect of $\mathrm{Al}$ content on the $[\mathrm{O}],[\mathrm{Mg}]$ and [Ca] under the condition of 50 mass $\% \mathrm{CaO}, 8$ mass $\% \mathrm{MgO}$, and other main chemical compositions in molten steel are shown in Table 5. In Fig. 6, as the $\mathrm{Al}$ content in steel increases from 0.0025 to 0.03 mass $\%$, the equilibrium [O] has a noticeable decrease when the $\mathrm{Al}$ content is below 0.015 mass $\%$, and almost keeps constant when the $\mathrm{Al}$ content is more than 0.015 mass $\%$. Hence, excessively high $\mathrm{Al}$ content in molten steel is unnecessary, and $0.01-0.02$ mass $\% \mathrm{Al}$ is permissible. Besides, the

Table 6. Chemical compositions of each steel sample, mass $\%$.

\begin{tabular}{ccccccccc}
\hline Number & $\mathrm{C}$ & $\mathrm{Si}$ & $\mathrm{Mn}$ & $\mathrm{Cr}$ & $\mathrm{Als}$ & $\mathrm{Ca}$ & $\mathrm{Mg}$ & $\mathrm{O}$ \\
\hline $\mathrm{A}$ & 0.98 & 0.24 & 0.33 & 1.48 & 0.020 & 0.0029 & 0.0009 & 0.0031 \\
$\mathrm{~B}$ & 0.98 & 0.24 & 0.34 & 1.52 & 0.021 & 0.0006 & 0.0003 & 0.0025 \\
$\mathrm{C}$ & 0.98 & 0.23 & 0.34 & 1.51 & 0.023 & 0.0028 & 0.0007 & 0.0021 \\
$\mathrm{D}$ & 0.99 & 0.24 & 0.34 & 1.55 & 0.033 & 0.0004 & 0.0006 & 0.0023 \\
E & 0.98 & 0.25 & 0.34 & 1.52 & 0.024 & 0.0015 & 0.0006 & 0.0030 \\
\hline
\end{tabular}

Table 7. Chemical compositions of each slag sample, mass $\%$.

\begin{tabular}{crrrrrr}
\hline Number & $\mathrm{CaO}$ & $\mathrm{SiO}_{2}$ & $\mathrm{MgO}$ & $\mathrm{Al}_{2} \mathrm{O}_{3}$ & $\mathrm{CaF}_{2}$ & Other \\
\hline A & 51.25 & 14.69 & 6.25 & 15.81 & 8.72 & 3.28 \\
B & 55.94 & 7.69 & 2.95 & 30.09 & 0.64 & 2.69 \\
C & 54.18 & 8.94 & 4.15 & 28.20 & 2.65 & 1.88 \\
D & 55.15 & 8.85 & 5.36 & 27.80 & 0.29 & 2.55 \\
E & 56.17 & 14.40 & 4.51 & 15.71 & 8.33 & 0.88
\end{tabular}

equilibrium $[\mathrm{Mg}]$ increases as the $\mathrm{Al}$ content increases, while with the increasing $\mathrm{Al}$ content, the equilibrium $[\mathrm{Mg}]$ almost keeps constant. Meanwhile, the equilibrium [Ca] increases noticeably as the $\mathrm{Al}$ content increases, which indicates that a high content of $\mathrm{Al}$ is more easy to react with $\mathrm{CaO}$, thus resulting in an increase in $\mathrm{CaO}$ inclusions.

Table 8. Calculated $[\mathrm{O}] \mathrm{s},[\mathrm{Mg}] \mathrm{s}$ and $[\mathrm{Ca}] \mathrm{s}$ contents of molten steel equilibrated with slags at the end of LF with $1833 \mathrm{~K}$.

\begin{tabular}{cccc}
\hline Number & Ca ppm & Mg ppm & O ppm \\
\hline A & 3.06 & 0.36 & 3.26 \\
B & 2.98 & 1.08 & 2.15 \\
C & 2.97 & 1.26 & 2.23 \\
D & 2.81 & 0.68 & 2.25 \\
E & 3.42 & 0.27 & 3.35 \\
\hline
\end{tabular}

Table 9. The number density (per $\mathrm{mm}^{2}$ ) of oxide inclusions in finished steel samples.

\begin{tabular}{cccc}
\hline Number & $\begin{array}{c}\text { Magnesia Alumina } \\
\text { Spinel }\end{array}$ & $\begin{array}{c}\text { Calcium Aluminate } \\
\text { Inclusion }\end{array}$ & $\begin{array}{c}\text { Number } \\
\text { Density }\end{array}$ \\
\hline 1 & 1.98 & 2.42 & 4.40 \\
2 & 2.49 & 1.25 & 3.74 \\
3 & 2.56 & 0.99 & 3.55 \\
4 & 2.23 & 0.97 & 3.20 \\
5 & 1.64 & 3.30 & 4.94 \\
\hline
\end{tabular}

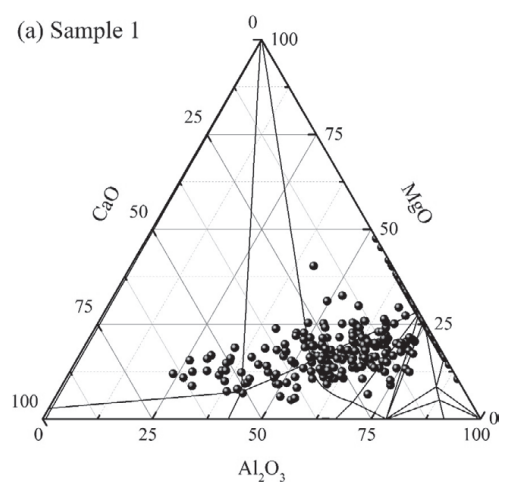

(a)

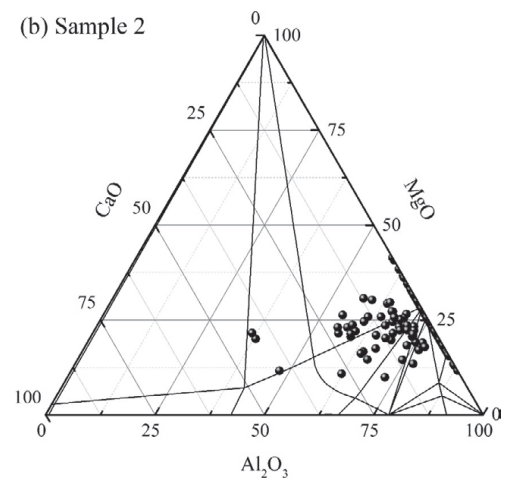

(b)

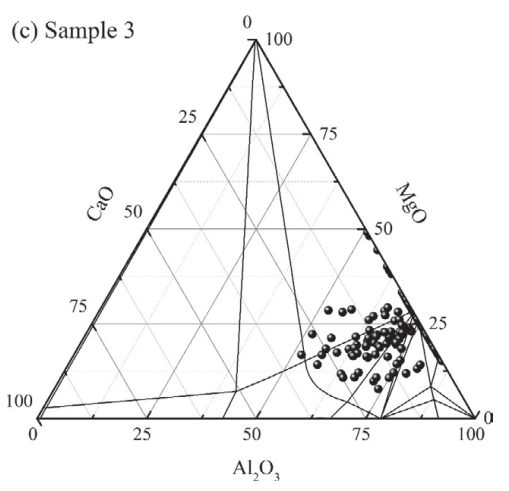

(c)

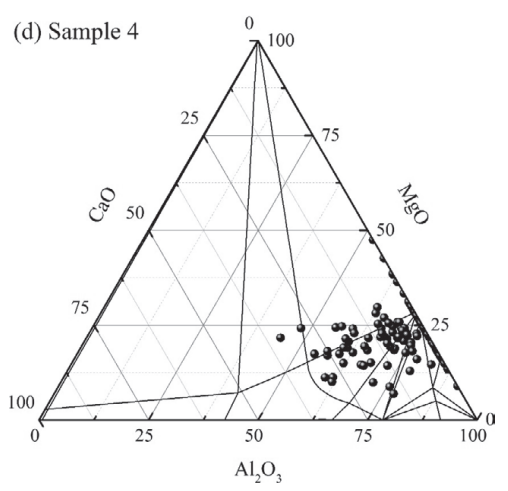

(d)

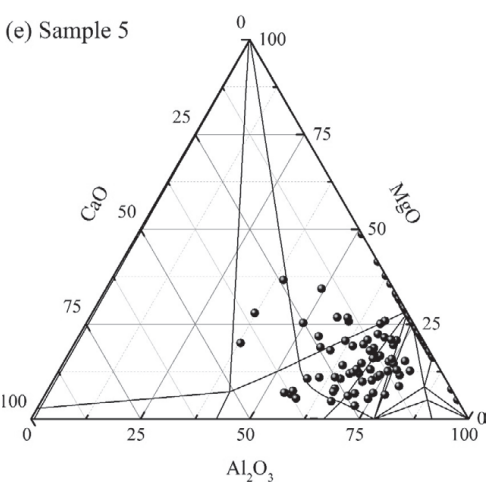

(e)

Fig. 10. Composition of oxide inclusions in each finished steel sample analyzed by INCA Feature Software, (a) Sample 1, (b) Sample 2, (c) Sample 3, (d) Sample 4, (e) Sample 5. 


\subsection{Effect of $(\mathrm{CaO})$ Content on Equilibrium $[\mathrm{O}],[\mathrm{Mg}]$ and $[\mathrm{Ca}]$}

Figure 7 displays the effect of $\mathrm{CaO}$ content on the [O], $[\mathrm{Mg}]$ and $[\mathrm{Ca}]$ under the condition of 0.015 mass\% $\mathrm{Al}, 8$ mass $\% \mathrm{MgO}$, and other main chemical compositions in molten steel are shown in Table 5. In Fig. 7, the [O] decreases but not obviously, while the $[\mathrm{Mg}]$ and $[\mathrm{Ca}]$ increases with an increase in the $\mathrm{CaO}$ content. Remarkably, the equilibrium $[\mathrm{Ca}]$ has a noticeable increase when the $\mathrm{CaO}$ content is increasing. This indicates that excessively high $\mathrm{CaO}$ is not advisable because globular calcium aluminates are more easily generated with high slag basicity. According to Fig. 7, $50-55$ mass $\% \mathrm{CaO}$ is permissible.

\subsection{Effect of (MgO) Content on Equilibrium [O], $[\mathrm{Mg}]$ and $[\mathrm{Ca}]$}

Figure 8 displays the effect of $\mathrm{MgO}$ content on the [O], $[\mathrm{Mg}]$ and $[\mathrm{Ca}]$ under the condition of 0.015 mass $\% \mathrm{Al}, 50$ mass $\% \mathrm{CaO}$, and other main chemical compositions in molten steel are shown in Table 5. In Fig. 8, as the $\mathrm{MgO}$ content in steel increases from 4 to 12 mass $\%$, the equilibrium [O] has a noticeable increase when the $\mathrm{MgO}$ content is below 10 mass $\%$, and almost keeps constant when the $\mathrm{MgO}$ content is more than 10 mass\%. Concerning about equilibrium [Ca] content, it decreases with an increase in the $\mathrm{MgO}$ content when the $\mathrm{MgO}$ content is below 9 mass\%, but increases with $\mathrm{MgO}$ when its content is more than 9 mass\%. This indicates that $8-10$ mass $\% \mathrm{MgO}$ is advisable under the calculation condition, because globular calcium aluminates are more difficult to generate with low equilibrium [Ca] content in molten steel.

\subsection{Effect of $\left(\mathrm{Al}_{2} \mathrm{O}_{3}\right)$ Content on Equilibrium $[\mathrm{O}],[\mathrm{Mg}]$ and $[\mathrm{Ca}]$}

Figure 9 shows the effect of $\mathrm{Al}_{2} \mathrm{O}_{3}$ content on the [O], $[\mathrm{Mg}]$ and $[\mathrm{Ca}]$ under the condition of 50 mass $\% \mathrm{CaO}, 8$ mass $\% \mathrm{MgO}$, and other main chemical compositions in molten steel are shown in Table 5. In Fig. 9, the equilibrium [O] almost keeps constant when the $\mathrm{Al}_{2} \mathrm{O}_{3}$ content is more than 25 mass $\%$. According to this figure, 20-30 mass $\% \mathrm{Al}_{2} \mathrm{O}_{3}$ is permissible to avoid the generation of globular calcium aluminates because of the increasing equilibrium $[\mathrm{Ca}]$ as the $\mathrm{Al}$ content in steel increases.

\subsection{Industrial Trials with the Application of This Model}

Industrial trials are carried out with the application of this model. In industrial production, $\mathrm{GCr} 15$ is produced by the process of a 30-ton electric arc furnace (EAF) melting $\rightarrow$ 30 -ton ladle furnace (LF) refining $\rightarrow 30$-ton vacuum degassing (VD) refining $\rightarrow$ ingot casting.

In order to validate the calculation results of this model, five industrial trials are carried out with different slag compositions. At the end of LF refining (1833 K), the steel and slag samples are taken together at the same time. After LF treatment, no alloy or flux is added, and the processing time of VD treatment and casting tempreture are essentially the same. Finally, the cleanliness of finished steel samples which are sampled at middle section of each ingots are examined by SEM. Meanwhile, the INCA Feature Software that is specifically developed for analysis and classification of steel inclusions is applied in this inclusion investigation.

The samples at the end of LF are numbered from A to E, and for finished steel, the samples are numbered from 1 to 5. Tables 6 and 7 respectively show the chemical composition of steel and slag samples at the sampling occasion. The chemical analyses are performed at the National Analysis

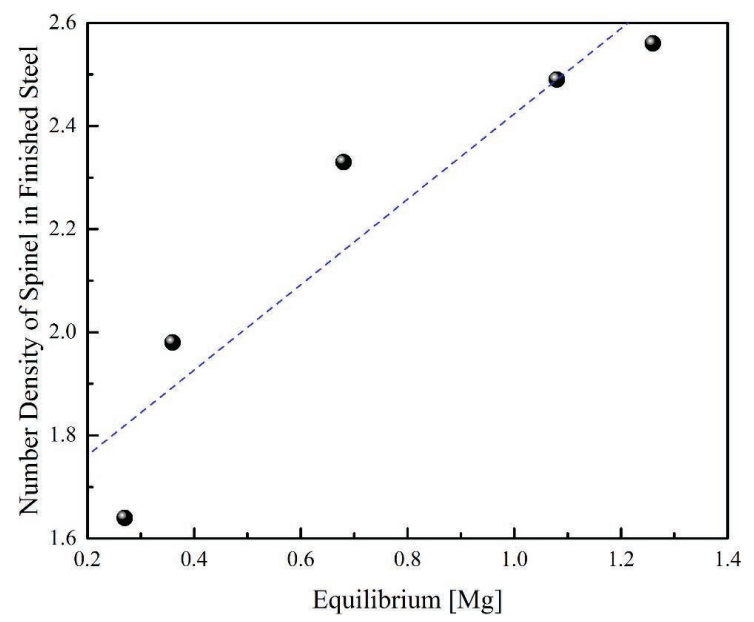

(a)

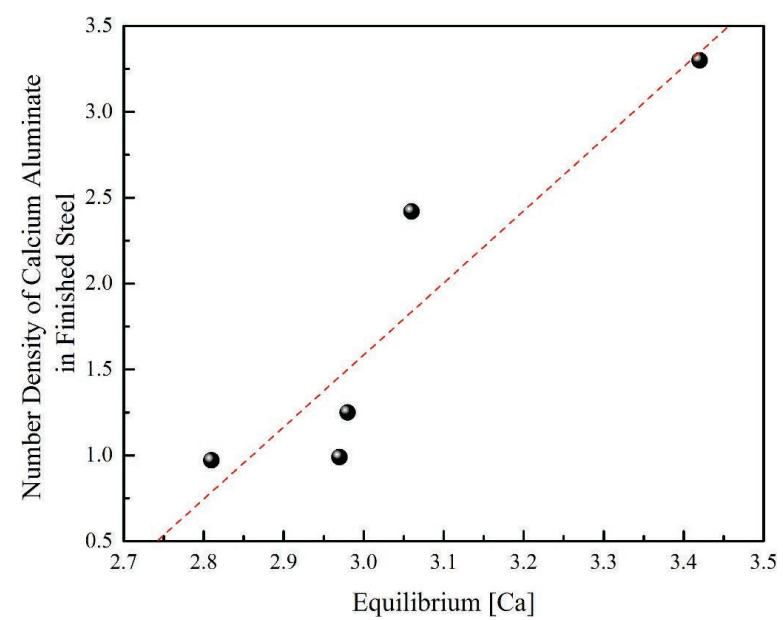

(b)

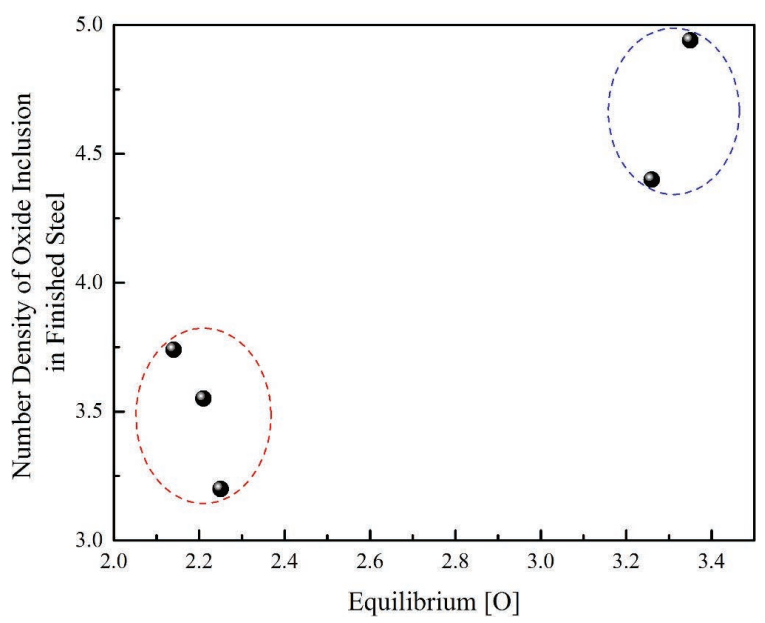

(c)

Fig. 11. Comparision between equilibrium content in molten steel and number density of oxide inclusion, (a) equilibrium $\mathrm{Mg}$ and spinel, (b) equilibrium $\mathrm{Ca}$ and calcium aluminate, (c) equilibrium $\mathrm{O}$ and oxide inclusion. (Online version in color.) 
Center for Iron and Steel (NACIS), China. The concrete methods of analyzing steel and slag are described in Ref. 33. In Table 6, the balance is Fe.

Table 8 show the calculated $[\mathrm{O}] \mathrm{s},[\mathrm{Mg}] \mathrm{s}$ and $[\mathrm{Ca}] \mathrm{s}$ contents of molten steel equilibrated with slags at the end of LF with the temperature of $1833 \mathrm{~K}$. According to Table 8, the calculated contents are much smaller than the measured contents. This is because these calculated contents are dissolved [O], [Ca] and $[\mathrm{Mg}]$, while the measured contents are total $[\mathrm{O}]$, [Ca] and [Mg].

The cleanliness of finished steel samples which are sampled at middle section of each ingots are examined by SEM and INCA Feature Software. The scaning area of each trials are $242 \mathrm{~mm}^{2}, 260.1 \mathrm{~mm}^{2}, 242 \mathrm{~mm}^{2}, 173.4 \mathrm{~mm}^{2}$ and $173.4 \mathrm{~mm}^{2}$, and the detection limit of inclusions diameter is $1 \mu \mathrm{m}$. The scanned oxide inclusions of each trial are shown in Table 9, and the oxide inclusion compositions are shown in Fig. 10. The oxide inclusions are divided into magnesia alumina spinel and calcium aluminate inclusion according to the $\mathrm{CaO}$ contents obtained by INCA software. If the $\mathrm{CaO}$ content is higher than 5 mass $\%$, it is considered as calcium aluminate. According to these figures, the $\mathrm{CaO}$ content in oxide inclusions are generally higher when the calculated [Ca]s is large, just as shown in Table 8.

Figure $\mathbf{1 1}$ is the number density of specific inclusion in finished steel versus the calculated equilibrium contents at the end of LF refining. In Figs. 10(a) and 10(b), it is shown that the number density of spinel inclusion is proportional to the calculated equilibrium $[\mathrm{Mg}]$ content, and the number density of calcium aluminate inclusion is proportional to the calculated equilibrium [Ca] content. Concerning the relationship between the number density of all oxide inclusion and the calculated equilibrium [O] content in Fig. 10(c), it is concluded that the number density of oxide inclusion is generally high if the calculated equilibrium [O] content is high. However, the relationship between the number density of oxide inclusion and equilibrium [O] content is no longer obvious when the equilibrium oxygen content is low. Overall, there is a good correlation between the calculated equilibrium contents and the number density of oxide inclusions, and the calculation results of this model have a good guidance for the production of high clean bearing steel.

\section{Conclusion}

(1) Given the contents $\mathrm{C}, \mathrm{Si}, \mathrm{Mn}, \mathrm{Cr}, \mathrm{Al}$ in molten steel, $\left(\mathrm{CaF}_{2}\right)$ and other two contents in $\mathrm{CaO}-\mathrm{SiO}_{2}-\mathrm{MgO}-$ $\mathrm{Al}_{2} \mathrm{O}_{3}-\mathrm{CaF}_{2}$ slag and temperature, the equilibrium slag composition and dissolved [O], $[\mathrm{Mg}]$ and [Ca] could be obtained quantitatively. This calculation model is verified by the comparison between the calculated results of this model and experimental data in other studies. It is shown that these two results are in good agreement, reflecting the reasonability of the model.

(2) The effects of $\mathrm{Al}$ in molten steel, $\mathrm{CaO}, \mathrm{MgO}$ and $\mathrm{Al}_{2} \mathrm{O}_{3}$ in slag on the equilibrium [O], $[\mathrm{Mg}]$ and $[\mathrm{Ca}]$ are discussed. The content of $\mathrm{Al}$ in steel should be controlled to an appropriate content, ranging from 0.01 to 0.02 mass $\%$. The proposed slag for GCr15 is listed as follows: 50-55 mass $\% \mathrm{CaO}, 8-10$ mass $\% \mathrm{MgO}, 20-30$ mass $\% \mathrm{Al}_{2} \mathrm{O}_{3}$, and the balance is $\mathrm{SiO}_{2}$ and $\mathrm{CaF}_{2}$.

(3) Industrial trials are conducted, and the composition of slag and steel at the end of LF refining and cleanliness of finished steel is investigated. Results show that there are good correlations between the calculated equilibrium contents and the number density of oxide inclusions. The number density of spinel and calcium aluminate inclusion increase with the increasing equilibrium $[\mathrm{Mg}]$ and $[\mathrm{Ca}]$ content calculated by this model respectly. This proves that the calculation results of this model have a good guidance for the production of high cleanliness bearing steel.

\section{Acknowledgements}

The authors would like to thank the National Natural Science Foundation of China (Grant No. 51674024, 51874034) for the financial support.

\section{REFERENCES}

1) K. Riyahimalayeri, P. Ölund and M. Selleby: Ironmaking Steelmaking, 40 (2013), 369.

2) G. Okuyama, K. Yamaguchi, S. Takeuchi and K. Sorimachi: ISIJ Int., 40 (2000), 121.

3) H. Ohta and H. Suito: Metall. Mater. Trans. B, 29 (1998), 119

4) G. M. Mehrotra, M. G. Frohberg, M. L. Kapoor and P. M. Mathew: Can. Metall. O., 13 (1974), 331.

5) X. Yang, J. Jiao, R. Ding, C. Shi and H. Guo: ISIJ Int., 49 (2009), 1828.

6) L. C. Oertel and A. Costa e Silva: Calphad, 23 (1999), 379.

7) B. H. Yoon, K. H. Heo, J. S. Kim and H. S. Sohn: Ironmaking Steelmaking, 29 (2002), 214.

8) I. H. Jung, S. A. Decterov and A. D. Pelton: Metall. Mater. Trans. $B, 35$ (2004), 493.

9) H. Wang, T. Ma and S. Wang: Trans. Tianjin Univ., 15 (2009), 187.

10) J. Xu, F. Huang and X. Wang: Metall. Mater. Trans. B, 47 (2016), 1217.

11) J. H. Park, D. S. Kim and S. B. Lee: Metall. Mater. Trans. B, 36 (2005), 67

12) H. Itoh, M. Hino and S. Ban-Ya: Metall. Mater. Trans. B, 28 (1997), 953.

13) J. Guo, S. Cheng, Z. Cheng and L. Xin: Steel Res. Int., 84 (2013), 545

14) F. Tsukihashi, E. Tawara and T. Hatta: Metall. Mater. Trans. B, 27 (1996), 967.

15) H. Itoh, M. Hino and S. Ban-Ya: Tetsu-to-Hagané, 84 (1998), 85 (in Japanese).

16) K. Hashimoto, T. Fujimatsu, N. Tsunekage, K. Hiraoka, K. Kida and E. C. Santos: Mater. Des., 32 (2011), 1605.

17) D. Bouchard and C. W. Bale: J. Phase Equilib., 16 (1995), 16.

18) A. D. Pelton and C. W. Bale: Metall. Trans. A, 17 (1986), 1211.

19) M. C. Heuzey and A. D. Pelton: Metall. Mater. Trans. B, 27 (1996), 810 .

20) J. Zhang and W. Yuan: J. Univ. Sci. Technol. Beijing, 17 (1995), 418.

21) J. Li and J. Zhang: J. Univ. Sci. Technol. Beijing, 22 (2000), 316.

22) X. Yang, J. Duan, C. Shi, M. Zhang, Y. Zhang and J. Wang: Metall. Mater. Trans. B, 42 (2011), 738.

23) X. Yang, C. Shi, M. Zhang, G. Chai and F. Wang: Metall. Mater. Trans. B, 42 (2011), 1150.

24) S. Duan, X. Guo, H. Guo and J. Guo: Ironmaking Steelmaking, 44 (2017), 168 .

25) S. Duan, C. Li, X. Guo, H. Guo, J. Guo and W. Yang: Ironmaking Steelmaking, 45 (2018), 655.

26) Verein Deutscher Eisenhüttenleute (VDEh) and M. Allibert: Slag Atlas, Woodhead Publishing, Düsseldorf, (1995), 39.

27) J. Zhang: Computational Thermodynamics of Metallurgical Melts and Solutions, The Publishing House of Metallurgical Industry, Beijing, (2007), 245.

28) X. Meng and G. Cheng: The 16th Annual Meeting of Steelmaking Institute (CSM 2016), ISIJ, Tokyo, (2016), 1025.

29) H. Suito, H. Inoue and R. Inoue: ISIJ Int., 31 (1991), 1381.

30) T. Kimura and H. Suito: Metall. Mater. Trans. B, 25 (1994), 33.

31) F. Tamura and H. Suito: Metall. Trans. B, 24 (1993), 121.

32) K. Riyahimalayeri, P. Ölund and M. Selleby: Steel Res. Int., 84 (2013), 136.

33) S. Li, G. Cheng, Z. Miao, L. Chen, C. Li and X. Jiang: ISIJ Int., 57 (2017), 2148. 